\title{
Potential Prognostic Significance of Patterns of Deletion (13q) in Plasma Cell Myelomas-Reappraisal of a Perennial Bone of Contention
}

\author{
Mohit Kumar Bhardwaj ${ }^{1 *}$ Sourav Kumar Mishra2* \\ Beklashwar Salona ${ }^{1}$ Sambit Kumar Mohanty ${ }^{1,3}$ \\ ${ }^{1}$ Department of Pathology and Laboratory Medicine, CORE \\ Diagnostics, Gurgaon, Haryana, India \\ ${ }^{2}$ Department of Medical Oncology, Advanced Medical Research \\ Institute, Bhubaneswar, Odisha, India \\ ${ }^{3}$ Department of Pathology and Laboratory Medicine, Advanced \\ Medical Research Institute, Bhubaneswar, Odisha, India
}

Shivani Sharma ${ }^{1}$

\begin{abstract}
Address for correspondence Sambit Kumar Mohanty, MD, FIAC, FACP, FRCPath, Department of Oncologic and Molecular Pathology, Advanced Medical Research Institute, \#1, Besides Satyasai Enclave, Khandagiri, Bhubaneswar, Odisha 751030, India

(e-mail: sambit04@gmail.com).
\end{abstract}

\begin{abstract}
Keywords

- myeloma

- deletion $13 q$

- prognosis

- interstitial

- terminal

Deletion $13 q$ is recommended in the initial cytogenetic workup of myeloma patients. The patterns of this abnormality have been shown to have differential prognostic value. The presence of monosomy 13 is associated with a significantly poor progression-free survival, while interstitial deletion $13 q$ is associated with significant improvement in the overall survival. We analyzed the patterns of $13 q$ abnormalities on fluorescent in situ hybridization (FISH) assay results in myeloma patients. Deletion $13 q$ abnormalities were observed in $38 \%$ ( 55 of 138 ) of the myeloma patients. Ten (18\%) and 44 (80\%) patients showed interstitial deletion and terminal deletion, respectively. One had a mosaic of both the patterns. Nine of the ten patients with interstitial deletions were males. For terminal deletion $13 \mathrm{q}$, there appeared to be a slight female predilection, with a male to female ratio of $0.83: 1$. Half of the patients with deletion $13 q$ had coexistent cytogenetic abnormalities. We suggest a baseline FISH for deletion $13 q$ and specification of the type of abnormality (terminal vs. interstitial) in patients with myeloma. Based on our observation in conjunction with the available literature, further studies in a large cohort of patients with survival data are warranted to clearly delineate the role of deletion $13 q$ in myeloma.
\end{abstract}

Plasma cell myeloma (PCM) is a clonal disorder of the bone marrow plasma cells. The initial cytogenetic workup includes detection of genomic abnormalities by fluorescent in situ hybridization (FISH) assay. Although the metaphase cytogenetics provide a global picture of the chromosomal aberrations, mitotic quiescence in the plasma cells compounded by low proliferation potential and complexity of the karyotype limits the use of this technique. Moreover, cryptic abnormalities and interstitial deletions cannot be detected by conventional cytogenetics. ${ }^{1}$ The National Comprehensive
Cancer Network (NCCN) guidelines recommend an interphase FISH panel on the bone marrow plasma cells for del (13q), $t(4 ; 14), t(14 ; 16), t(11 ; 14), t(14 ; 20)$, del (17p), 1q amplification, and del (1q) during the initial evaluation of PCM. ${ }^{1}$ NCCN as well as the Mayo clinic risk stratification for multiple myeloma (mSMART) lists $\mathrm{t}(4 ; 14), \mathrm{t}(14 ; 16)$, $t(14 ; 20)$, del (17p), and 1q gain in the high-risk category. ${ }^{2}$ Del (13q) and other genetic abnormalities are grouped under the standard risk category. ${ }^{2}$ However, based on the previous and recent data, there is ambiguity pending further
Dol https://doi.org/ $10.1055 / \mathrm{s}-0041-1732852$ ISSN 0971-5851 (c) 2021. Indian Society of Medical and Paediatric Oncology. This is an open access article published by Thieme under the terms of the Creative Commons Attribution-NonDerivative-NonCommercial-License, permitting copying and reproduction so long as the original work is given appropriate credit. Contents may not be used for commercial purposes, or adapted, remixed, transformed or built upon. (https://creativecommons.org/licenses/by-nc-nd/4.0/).

Thieme Medical and Scientific Publishers Private Ltd. A-12, Second Floor, Sector -2, NOIDA -201301, India 
clarification on the role of del (13q) in PCM..$^{3-7}$ That being the case, whether $13 \mathrm{q}$ abnormalities should at all be assessed in the initial workup of myeloma, and if included what should be the FISH reporting pattern(s) of this aberration, including their possible relevance.

Herein, we attempted to address the above-mentioned issue based on the FISH results from our center. This is a retrospective analysis, following approval from the Ethics Committee Board, CORE Diagnostics, Gurgaon, Haryana, India (CORE_IRB_001) Institutional review board, and did not have any impact on the patient care. The procedures followed were in accordance with the ethical standards of the responsible committee on human experimentation (institutional or regional) and with the Helsinki Declaration of 1964, as revised in 2013. The cases of PCM with del (13q) in isolation or in conjunction with other abnormality were retrieved from the electronic database. All the saved FISH images were further analyzed independently by three molecular pathologists (MB, SS, and SKM) and a consensus on the type of deletion was made. The study period was from January 2017 to December 2018. As part of the evaluation of PCM, we typically conduct FISH testing to identify del (13q), $\mathrm{t}(4 ; 14), \mathrm{t}(14 ; 16), \mathrm{t}(11 ; 14), \mathrm{t}(14 ; 20)$, and del (17p) following plasma cell enrichment by adding anti-CD138 monoclonal antibody-coated magnetic beads. For del (13q), we use locus specific dual color (LSP) FISH probe to detect the deletions in the 13q14.2 (RB1) and 13q34 (LAMP1), respectively. Other probes used in our laboratory include [LSP (TP53/CCP17) and locus specific dual color and dual fusion probes including IgH/FGFR1, IgH/CCND1, IgH/MAF, and $\operatorname{IgH} / M A F B]$ for $\operatorname{del}(17 \mathrm{p})$, and IgH- related translocations (Cytotest Inc., Rockville, Maryland, United States). FISH assay was performed based on the manufacturer's protocol. ${ }^{8}$ A total of 200 interphase plasma cell nuclei were evaluated. The cutoff used for positivity with del (13q) and del (17p) is 6\%, and the cutoff for the dual fusion translocation probes is $15 \%$.
Del (13q) abnormalities were detected in 55 (38\%) of the 145 patients studied. The median age for the positive cases was 62 years (range $=35-90$ years), and a three quarter of them were above 55 years. Of these 55 positive cases, 10 (18\%) showed interstitial deletion (-Fig. 1A), 44 (80\%) had terminal deletion/monosomy13 ( - Fig. 1B), and one was a mosaic of both the patterns. Nine of the ten patients with interstitial deletions were males denoting a male preponderance and could be an incidental finding. For terminal deletion 13, there appeared to be a slight female predilection, with a male to female ratio of $0.83: 1$. Half of the patients with $13 q$ deletions had coexistent cytogenetic abnormalities. While 85\% (23 of $27)$ of the patients with combined abnormalities [del(17p) $(n=5) ; \mathrm{t}(4 ; 14)(n=7) ; \operatorname{del}(17 \mathrm{p})$ and $\mathrm{t}(4 ; 14)(n=3) ; \mathrm{t}(11 ; 14)$ $(n=5) ; \mathrm{t}(14 ; 16)(n=3)]$ had terminal deletions, only $15 \%$ (4 of 10) had interstitial deletions $[\operatorname{del}(17 \mathrm{p})(n=1) ; \mathrm{t}(4 ; 14)$ $(n=3)]$ (-Table 1).

In the natural history of PCM, trisomies and immunoglobulin heavy-chain translocations are the initial events or the first hit in tumorigenesis. ${ }^{2}$ In the transition from a monoclonal gammopathy of unknown significance (MGUS) to PCM, secondary molecular events or second hit are accrued in the neoplastic plasma cells. ${ }^{2}$ In spite of the presence of del (13q) in a large proportion of PCM patients and its relative rarity in MGUS, its role in the pathogenesis is unclear. ${ }^{3,9}$ It is possibly a part of the second hit in the oncogenesis of myeloma cells. Most deletions are localized to the 13q14 locus that harbors the retinoblastoma $(R B)$ gene. ${ }^{10-12} R B$ belongs to the family of tumor suppressor genes. Heterozygous deletion of the $R B$ gene is a common event in the myeloma cells with inconsequential effect on the downstream gene transcription and protein expression. It has been observed that many components of the $R B$ pathway such as cyclin D, p16, and E2F are dysregulated in neoplastic plasma cells. ${ }^{10-12}$ Mutations in the "disrupted in B-cell malignancy" tumor suppressor gene, located $530 \mathrm{~kb}$ telomeric to the $R B$ gene locus, may render
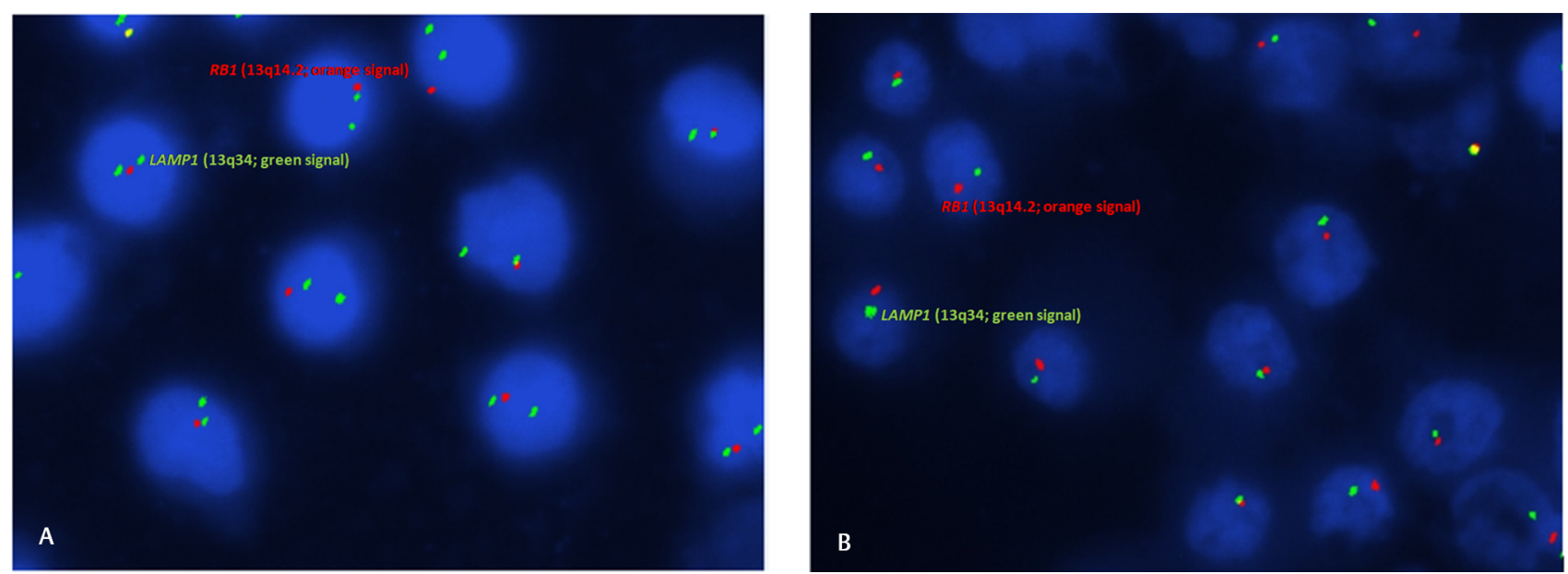

Fig. 1 (A) Locus specific probe (LSP) for RB1 (13q14.2; orange signal) and LSP for LAMP1 (13q34; green signal) on the interphase cells showing interstitial del(13q)/loss of $R B 1$ gene as indicated by one orange and two green signals (fluorescent in situ hybridization [FISH] image at $60 \mathrm{x}$ magnification; Olympus 61 microscope using Bioview software, Israel). (B) LSP forRB1 (13q14.2; orange signal) and LSP for LAMP1 (13q34; green signal) on the interphase cells showing terminal del(13q)/loss of RB1 and LAMP1genes as indicated by one orange and one green signals (FISH image at 60x magnification; Olympus 61 microscope using Bioview software). 
Table 1 Patterns of del (13q) in plasma cell myeloma cases

\begin{tabular}{|l|l|l|l|l|l|}
\hline Types of del(13q) & Mean age & Gender (M:F) & Other abnormalities & $\geq 55$ years & $<55$ years \\
\hline $\begin{array}{l}\text { Interstitial deletion } 10 \\
(18.18 \%)\end{array}$ & 61.3 & $9: 1$ & $4(14.8 \%)$ & $7(70 \%)$ & $3(30 \%)$ \\
\hline $\begin{array}{l}\text { Terminal deletion } 44 \\
(80 \%)\end{array}$ & 60.77 & $0.83: 1$ & $23(85.18 \%)$ & $34(77.27 \%)$ & $10(22.72 \%)$ \\
\hline
\end{tabular}

plasma cells resistant to apoptosis. Since the $R B$ suppresses interleukin 6 (IL-6) production and secretion, its deletion may result in the dysregulation of IL-6 expression and hence expansion of IL-6 dependent myeloma clones. ${ }^{3}$

Except for one study, which reported del (13q) in $86 \%$ of their FISH samples, others have reported this abnormality in 30 to $50 \%$ of the patients. ${ }^{13,14}$ There was an abstract from India with limited number of cases that showed 15\% incidence of del $(13 q) \cdot{ }^{15}$ Deletions in the long arm of chromosome 13 can be either interstitial or terminal. Monosomy 13 is the commonest $13 \mathrm{q}$ abnormality in myeloma and accounts for 85 to $90 \%$ of all $13 q$ abnormalities across multiple studies. ${ }^{3-7}$ Interstitial deletions were reported in 6 to $15 \%$ patients and were usually large deletions spanning across the $13 q 14$ till the telomeric end of the $q$ arm.,13

The sensitivity of conventional metaphase cytogenetics in detecting del (13q) ranges between 30 and 50\% and hence 50 to $70 \%$ of these deletions can be missed. ${ }^{4,6}$ This results from the low proliferative index of the neoplastic plasma cells and can be resolved with interphase FISH studies. There appears to be a high degree of concordance between del (13q) detected on conventional cytogenetics and FISH.

Though not considered as a high-risk abnormality, there is ample controversy regarding the prognostic implication of del (13q) in PCM. Based on studies conducted in the 90s, it was reported that partial or total loss of $13 q$ was an independent and one of the most important parameters associated with poor prognosis in patients with myeloma. ${ }^{16}$ Subsequently, it was observed that del (13q) was associated with poor prognosis, only if detected by conventional cytogenetics and not by FISH. ${ }^{17}$ Majority of these $\operatorname{del}(13 q)$ were terminal deletions and hence there appeared to be no prognostic value in triaging sample for FISH to detect interstitial del(13q). In other studies, del (13q) was an independent marker for poor prognosis on univariate analysis, but not so on multivariate regression analysis. ${ }^{18,19}$ These observations led to the conclusion that del (13q) as a standalone marker was not associated with adverse outcomes, rather the poor prognosis was due to cosegregation of other high-risk cytogenetic abnormalities such as $\operatorname{del}(4 ; 14)$ and $\operatorname{del}(17 \mathrm{p}){ }^{5}$

In this context, the analysis by Binder et al sheds some light on the prognostic implications of del (13q) in patients with myeloma. ${ }^{7}$ Of the 1181 patients analyzed by FISH and conventional cytogenetics, a del (13q) abnormality was seen in $42 \%$ patients. There were 411 (35\%) patients with monosomy 13, 73 (6\%) with interstitial deletions, and 9 (1\%) with a mosaic pattern. The presence of monosomy 13 (compared with its absence) was associated with a significantly poor progression-free survival (median PFS: 1.75 vs. 2.00 years, $p=0.013$ ) as well as overall survival (median OS:
5 vs. 8.3 years, $p<0.001)$. In contrast, the presence of interstitial del (13q) (in comparison to its absence) had a salutary effect on the prognosis with significant improvement in OS (median not reached vs. 6.4 years, $p=0.006$ ) without an impact on the PFS (median: 1.93 vs. 1.66 years, $p=0.314$ ). Binder at al also highlighted that segregation of the $13 q$ abnormality into interstitial or terminal deletions had impact on the prognosis of del (13q) in myelomas. ${ }^{7}$ Interestingly, the prognosis associated with these abnormalities was retained even after adjusting for age, gender, international staging system stage, first-line therapy, and the presence of the established high-risk cytogenetic abnormalities. ${ }^{7}$ This is the first study demonstrating the contrasting effects of patterns of del $(13 q)$ on the outcome of patients treated for myeloma. So, there appears to be a prognostic dichotomy regarding the differential effect of patterns of del (13q) in the myeloma patients as observed in the above ${ }^{7}$ and prior studies. First of all, chromosome 13 abnormalities have been analyzed as a single entity throughout and not as two different patterns, that is, monosomy and del (13q) as detected on FISH. Second, considering the small number of patients with interstitial deletions, most studies lacked sufficient power to detect an impact on survival. Lastly, almost all of these studies predated the introduction of novel antimyeloma drugs and may not have been able to detect their differential effect on specific cytogenetic abnormalities. ${ }^{20}$

In conclusion, we recommend a baseline FISH for del (13q) including the pattern of abnormality (terminal vs. interstitial) in addition to karyotyping in patients with PCM. Based on our observation in conjunction with the available literature, further studies in a large cohort of patients are warranted to clearly delineate the prognostic value of del (13q) in PCMs.

\section{Note}

This abstract was presented as a poster at the American Molecular Pathology Society Annual Meeting, 2018 in San Antonio, Texas.

\section{Disclosure of Funding/Support \\ None.}

\section{Conflict of Interest}

The authors have stated that they have no conflicts of interest.

\section{References}

1 NCCN guidelines for multiple myeloma [ https://www.nccn. org/professionals/physician_gls/pdf/myeloma.pdf]. Accessed June 30, 2021 
2 Rajkumar SV. Multiple myeloma: 2020 update on diagnosis, risk-stratification and management. Am J Hematol 2020;95(5):548-567

3 Fonseca R, Oken MM, Harrington D, et al. Deletions of chromosome 13 in multiple myeloma identified by interphase FISH usually denote large deletions of the $\mathrm{q}$ arm or monosomy. Leukemia 2001;15(6):981-986

4 Avet-Louseau H, Daviet A, Sauner S, Bataille R; Intergroupe Francophone du Myélome. Chromosome 13 abnormalities in multiple myeloma are mostly monosomy 13 . Br J Haematol 2000;111(4):1116-1117

5 Avet-Loiseau H, Attal M, Moreau P, et al. Genetic abnormalities and survival in multiple myeloma: the experience of the Intergroupe Francophone du Myélome. Blood 2007;109(8):3489-3495

6 Zojer N, Königsberg R, Ackermann J, et al. Deletion of 13q14 remains an independent adverse prognostic variable in multiple myeloma despite its frequent detection by interphase fluorescence in situ hybridization. Blood 2000;95(6):1925-1930

7 Binder M, Rajkumar SV, Ketterling RP, et al. Prognostic implications of abnormalities of chromosome 13 and the presence of multiple cytogenetic high-risk abnormalities in newly diagnosed multiple myeloma. Blood Cancer J 2017;7(9):e600-e600

8 Wiktor AE, Van Dyke DL, Stupca PJ, et al. Preclinical validation of fluorescence in situ hybridization assays for clinical practice. Genet Med 2006;8(1):16-23

9 Avet-Loiseau H, Li JY, Morineau N, et al. Monosomy 13 is associated with the transition of monoclonal gammopathy of undetermined significance to multiple myeloma. Intergroupe Francophone du Myélome. Blood 1999;94(8):2583-2589

10 Juge-Morineau N, Harousseau JL, Amiot M, Bataille R. The retinoblastoma susceptibility gene RB-1 in multiple myeloma. Leuk Lymphoma 1997;24(3-4):229-237

11 Chng WJ, Glebov O, Bergsagel PL, Kuehl WM. Genetic events in the pathogenesis of multiple myeloma. Best Pract Res Clin Haematol 2007;20(4):571-596

12 Dao DD, Sawyer JR, Epstein J, Hoover RG, Barlogie B, Tricot G. Deletion of the retinoblastoma gene in multiple myeloma. Leukemia 1994;8(8):1280-1284
13 Shaughnessy J, Tian E, Sawyer J, et al. High incidence of chromosome 13 deletion in multiple myeloma detected by multiprobe interphase FISH. Blood 2000;96(4):1505-1511

14 Kadam Amare PS, Jain H, Nikalje S, et al. Observation on frequency \& clinico-pathological significance of various cytogenetic risk groups in multiple myeloma: an experience from India. Indian J Med Res 2016;144(4):536-543

15 Dhiman P, Goel S, Samal P, et al. FISH Analysis in Multiple Myeloma - a Retrospective Study from India. Blood 2016;128(22):5646

16 Tricot G, Barlogie B, Jagannath S, et al. Poor prognosis in multiple myeloma is associated only with partial or complete deletions of chromosome 13 or abnormalities involving $11 \mathrm{q}$ and not with other karyotype abnormalities. Blood 1995;86(11):4250-4256

17 Chiecchio L, Protheroe RKM, Ibrahim AH, et al. Deletion of chromosome 13 detected by conventional cytogenetics is a critical prognostic factor in myeloma. Leukemia 2006;20(9):1610-1617

18 Gutiérrez NC, Castellanos MV, Martín ML, et al. GEM/ PETHEMA Spanish Group. Prognostic and biological implications of genetic abnormalities in multiple myeloma undergoing autologous stem cell transplantation: $\mathrm{t}(4 ; 14)$ is the most relevant adverse prognostic factor, whereas RB deletion as a unique abnormality is not associated with adverse prognosis. Leukemia 2007;21(1):143-150

19 Boyd KD, Ross FM, Chiecchio L, et al. NCRI Haematology Oncology Studies Group. A novel prognostic model in myeloma based on co-segregating adverse FISH lesions and the ISS: analysis of patients treated in the MRC Myeloma IX trial. Leukemia 2012;26(2):349-355

20 Binder M, Rajkumar SV, Gertz MA, et al. Predictors of early response to initial therapy in patients with newly diagnosed symptomatic multiple myeloma. Am J Hematol 2015;90(10):888-891 